\title{
Correction to: The EURO-FORTA (Fit fOR The Aged) List: International Consensus Validation of a Clinical Tool for Improved Drug Treatment in Older People
}

\author{
Farhad Pazan $^{1} \cdot$ Christel Weiss $^{2} \cdot$ Martin Wehling $^{1} \cdot$ FORTA
}

Published online: 2 July 2018

(c) Springer International Publishing AG, part of Springer Nature 2018

\section{Correction to: Drugs Aging (2018) 35(1):61-71 https://doi.org/10.1007/s40266-017-0514-2}

In the original publication of this article, the members of the FORTA group were provided in such a way that they could not be indexed as collaborators on PubMed. The article should have included an Acknowledgement section as shown below.

Acknowledgements The FORTA group consists of the following members: Burkhardt H, Schwarz S, Wedding U, Johnell K, Eriksson T, Ruths S, Husebø BS, Huupponen R, Ranhoff AH, Onder G, Incalzi RA, Marcucci M, Cherubini A, Volpato S, Ruggiero C, Mugelli A, Laville M, Annweiler C, Mouchoux C, Robert P, Rhalimi M, Solano Jaurrieta
JJ, Rodríguez Mañas L, Macías Núñez JF, Cruz Jentoft AJ, Serra Rexach JA, Agustí A, Mateos R, San-José Laporte A, Gladman J, Crome P, Hughes C, O'Mahony D, Byrne S, Ekpo E, Ashton C, Witham MD, Soiza RL, Wieczorowska-Tobis K, Kostka T, Sławek J, Klich-Rączka A, Grodzicki T, Bień B, Broncel M, Mierzejewski P, Jansen RWMM, van den Bemt PMLA, van der Cammen TJM, Bauer J, Berthold HK, Denkinger M, Dovjak P, Frohnhofen H, Frühwald T, Gisinger C, Gogol M, Gosch M, Gutzmann H, Heuser I, Hüll M, Iglseder B, Kasper S, Kwetkat A, von Renteln-Kruse W, Schindler C, Schuler M, Schulz RJ, Sommeregger U, Zeyfang A.
The members of FORTA are listed in Acknowledgements.

The original article can be found online at https://doi.org/10.1007/ s40266-017-0514-2.

Martin Wehling

martin.wehling@medma.uni-heidelberg.de

1 Institute for Experimental and Clinical Pharmacology and Toxicology, Clinical Pharmacology, Medical Faculty

Mannheim, Ruprecht-Karls-University Heidelberg,

Mannheim, Germany

2 Department of Medical Statistics, Biomathematics and Information Processing, Medical Faculty Mannheim, Heidelberg University, Mannheim, Germany 